\title{
EFFECTS OF A SPEED OF PROCESSING INTERVENTION ON DRIVING PERFORMANCE: THE ACCELERATE STUDY
}

\author{
Karlene Ball, David Ball, Meredith Rumble, David Edwards, Virginia Wadley \\ University of Alabama at Birmingham \\ Department of Psychology and Center for Research on Applied Gerontology
}

Useful Field of View performance, as measured by UFOV ${ }^{\circledR}$, is a valid and reliable predictor of crash involvement among older drivers, and UFOV ${ }^{\circledR}$ performance improves with Speed of Processing (SOP) training. The ACCELERATE Study is examining the effects of SOP training on other cognitive functions and on everyday mobility among older adults at risk for impaired mobility.

To date, 59 participants have been randomly assigned to SOP training and 59 to an Internet training control group (total $n=118$ ). At baseline and post-test, participants are given extensive cognitive, sensory, health, and mobility assessments, as well as driving assessments in either a driving simulator or an instrumented vehicle.

Preliminary results indicate that performance on the UFOV ${ }^{\circledR}$ improves significantly more in the SOP training group than in the control group. Furthermore, means on most other cognitive variables are in the direction of greater improvement for SOP trainees than controls, with significant transfer of training on select speeded measures.

Preliminary analyses of driving indicate that relative to controls, SOP trainees have improved in the speed with which they are able to detect moving targets originating in the periphery and moving toward central vision, but not in the detection of static targets originating in central view. Thus, some driving tasks appear to benefit from SOP training, while others do not.

Results suggest that Speed of Processing training may transfer to other cognitive functions as well as to everyday mobility performance, such as driving. 